\title{
Charge transfer excitations, pair density waves, and superconductivity in moiré materials
}

\author{
Kevin Slagle $\circledast^{1,2}$ and Liang $\mathrm{Fu}^{3}$ \\ ${ }^{1}$ Department of Physics and Institute for Quantum Information and Matter, California Institute of Technology, \\ Pasadena, California 91125, USA \\ ${ }^{2}$ Walter Burke Institute for Theoretical Physics, California Institute of Technology, Pasadena, California 91125, USA \\ ${ }^{3}$ Department of Physics, Massachusetts Institute of Technology, Cambridge, Massachusetts 02139, USA
}

(Received 7 July 2020; accepted 8 December 2020; published 21 December 2020)

\begin{abstract}
Transition-metal dichalcogenide (TMD) bilayers are a new class of tunable moiré systems attracting interest as quantum simulators of strongly interacting electrons in two dimensions. In particular, recent theory predicts that the correlated insulator observed in $\mathrm{WSe}_{2} / \mathrm{WS}_{2}$ at half filling is a charge-transfer insulator similar to cuprates and, upon further hole doping, exhibits a transfer of charge from anionlike to cationlike orbitals at different locations in the moiré unit cell. In this work, we demonstrate that in this doped charge-transfer insulator, tightly bound charge- $2 e$ excitations can form to lower the total electrostatic repulsion. This composite excitation, which we dub a trimer, consists of a pair of holes bound to a charge-transfer exciton. When the bandwidth of doped holes is small, trimers crystallize into insulating pair density waves at a sequence of commensurate doping levels. When the bandwidth becomes comparable to the pair binding energy, itinerant holes and charge- $2 e$ trimers interact resonantly, leading to unconventional superconductivity similar to superfluidity in an ultracold Fermi gas near Feshbach resonance. Our theory is broadly applicable to strongly interacting charge-transfer insulators, such as $\mathrm{WSe}_{2} / \mathrm{WS}_{2}$ or TMD homobilayers under an applied electric field.
\end{abstract}

DOI: 10.1103/PhysRevB.102.235423

Moiré superlattices [1-3] can be viewed as magnified crystals whose unit cell is of nanometer instead of angstrom size. Correspondingly, the relevant electronic phenomena in moiré superlattices is governed by the coarse-grained moiré potential and the extended Coulomb repulsion, with characteristic energy scale on the order of meV instead of eV. Thanks to the increase of length scale and the reduction of energy scale, moiré systems feature remarkable tunability through the control of twist angle and displacement field. A variety of moiré materials have emerged as exciting venues for studying and designing correlated electron phenomena with an unprecedented level of control [4-15].

Recently, moiré superlattices of transition-metal dichalcogenide (TMD) layers [16] have attracted great interest as quantum simulators of strongly correlated electron systems in two dimensions [17-22]. By varying the twist angle, the relative strength of the bandwidth and electron interaction can be tuned, and a rich quantum phase diagram can potentially be realized [23-26]. Encouragingly, transport and optical experiments are starting to observe correlated insulating states in the TMD heterobilayer $\mathrm{WSe}_{2} / \mathrm{WS}_{2}$ with $n \leqslant 1$ holes per moiré unit cell [27-29]. In particular, the insulating state at $n=1$ is theoretically identified as a charge-transfer insulator with a cation and an anion at different locations in the moiré unit cell, corresponding to localized Wannier orbitals at the primary and secondary energy minimum of the moiré potential, respectively $[30,31]$. While a charge-transfer insulator is similar to a Mott insulator in terms of ground-state properties, the key difference is that, upon doping a charge-transfer insulator to $n>1$, the additional charges fill a higher-energy orbital in order to avoid double occupancy [32]. A famous example of charge-transfer insulators is undoped cuprates [33,34], for which a link between charge-transfer physics and hightemperature superconductivity upon doping has long been proposed and studied [35-37].

In this work, we present a microscopic theory of charge pairing by Coulomb repulsion in TMD heterobilayers under a range of fillings $n>1$. This counterintuitive phenomenon occurs when the charge-transfer gap at $n=1$ is small, so that two doped charges can lower their energy by polarizing their surroundings to form a tightly bound charge- $2 e$ "trimer" that consists of three holes on adjacent cations surrounding an electron on an anion. We show that the trimer costs less energy than two spatially separated holes for realistic forms of electron-electron interaction. When the single-particle bandwidth is small, we predict the formation of periodic density waves of trimers at certain doping levels $n=1+p / q>1$ ( $p, q$ are integers), whose periodicity is commensurate with the moiré lattice. As the bandwidth of holes increases and becomes comparable with the binding energy of trimers, holes and trimers coexist and interact resonantly to form a strongcoupling superconductor, similar to a strongly interacting superfluid in a Fermi gas near Feshbach resonance [38,39]. Our theory of pair density waves and superconductivity in TMD heterobilayers is asymptotically exact in a certain regime of strong interaction and small doping.

We start by describing the single-particle electronic structure of TMD heterobilayers (e.g., $\mathrm{WSe}_{2} / \mathrm{WS}_{2}$ ). Here, the topmost valence band of $\mathrm{WSe}_{2}$ is reconstructed into a set of moiré bands by the periodic moiré potential resulting from 
the $4 \%$ lattice mismatch with $\mathrm{WS}_{2}$. Importantly, the moiré potential has two inequivalent local minima located at the $A A$ and $A B$ stacking regions, giving rise to two sets of localized Wannier orbitals. The $A A(A B)$ orbital has a lower (higher) on-site energy for holes and can be regarded as anionlike (cationlike).

Thus, the low-energy physics of a TMD heterobilayer can be faithfully mapped onto a two-dimensional diatomic crystal with one cation and one anion per unit cell. The effective Hamiltonian takes the form of an extended Hubbard model on the honeycomb lattice [30]:

$$
\begin{aligned}
H & =H_{K}+H_{0}, \quad H_{K}=\sum_{i j, s=\uparrow \downarrow} t_{i j} c_{i s}^{\dagger} c_{j s}, \\
H_{0} & =\sum_{j \in B} \Delta n_{j}+\sum_{i} U n_{i \uparrow} n_{i \downarrow}+\frac{1}{2} \sum_{i \neq j} V_{i j} n_{i} n_{j},
\end{aligned}
$$

where the A and B sublattices (colored black and red in the figures) correspond to the anion and cation, respectively. $c_{i}^{\dagger}$ creates a hole in the moiré valence band with charge $e>0$, and $n_{i}=n_{i \uparrow}+n_{i \downarrow} . \Delta>0$ is the energy difference of cation and anion orbitals. For $\mathrm{WSe}_{2} / \mathrm{WS}_{2}, \Delta=$ $14.9 \mathrm{meV}$ is extracted from first-principles band-structure calculations $[30,40]$.

\section{TRIMERS}

The hopping integrals $t_{i j}$ decrease exponentially as the moiré period $L_{\mathrm{M}}$ increases. The on-site and extended twobody interactions $U, V_{i j}>0$ are given by Coulomb integrals in the Wannier basis [30,40]. Since $U$ and $V_{i j}$ decrease as power-law functions of $L_{\mathrm{M}}$, electron-electron interactions dominate over single-particle hopping at large $L_{\mathrm{M}}$. In this strong-coupling regime, the on-site repulsion $U$ is the largest relevant energy scale. At the filling $n=1$, the system is in an insulating state where all anions are singly occupied and cations unoccupied; i.e., $n_{i}^{0}=1$ for $i \in A$ and $n_{i}^{0}=0$ for $i \in B$.

Upon doping to $n>1$, the additional $n-1$ charges have to occupy the cations in order to avoid the large energy cost of double occupancy. To study the many-body physics at finite doping, we first identify the relevant charged excitations at $n=1$. For this purpose, it is useful to rewrite $H_{0}$ in terms of $\delta n_{i} \equiv n_{i}-n_{i}^{0}$, the change of occupation relative to the ground state:

$$
H_{0}=\sum_{i \in A} V_{a} \delta n_{i}+\sum_{j \in B}\left(\Delta+V_{c}\right) \delta n_{j}+\frac{1}{2} \sum_{i \neq j} V_{i j} \delta n_{i} \delta n_{j} .
$$

We have taken the $U=\infty$ limit so that double occupancy is forbidden. Then, there exist two types of elementary excitations: (1) electrons on the $A$ sublattice of anions with $\delta n=-1$ (charge $-e$ ), and (2) holes on the $B$ sublattice of cations with $\delta n=1$ (charge $+e) . V_{c}\left(V_{a}\right)$ is the self-energy of a hole (electron) due to its electrostatic interaction with all other charges in the ground state, defined by $V_{c}\left(V_{a}\right)=\sum_{j \in A} V_{i j}$ with $i \in$ $B(A)$ [41]. The Coulomb energies $V_{i j}$ decrease rapidly due to screening effects when the distance between sites $i$ and $j$ exceeds the distance to nearby metallic gates.

It follows from Eq. (2) that adding a hole to the chargetransfer insulator costs energy $E_{e}-\mu$, where $E_{e}=\Delta+V_{c}$
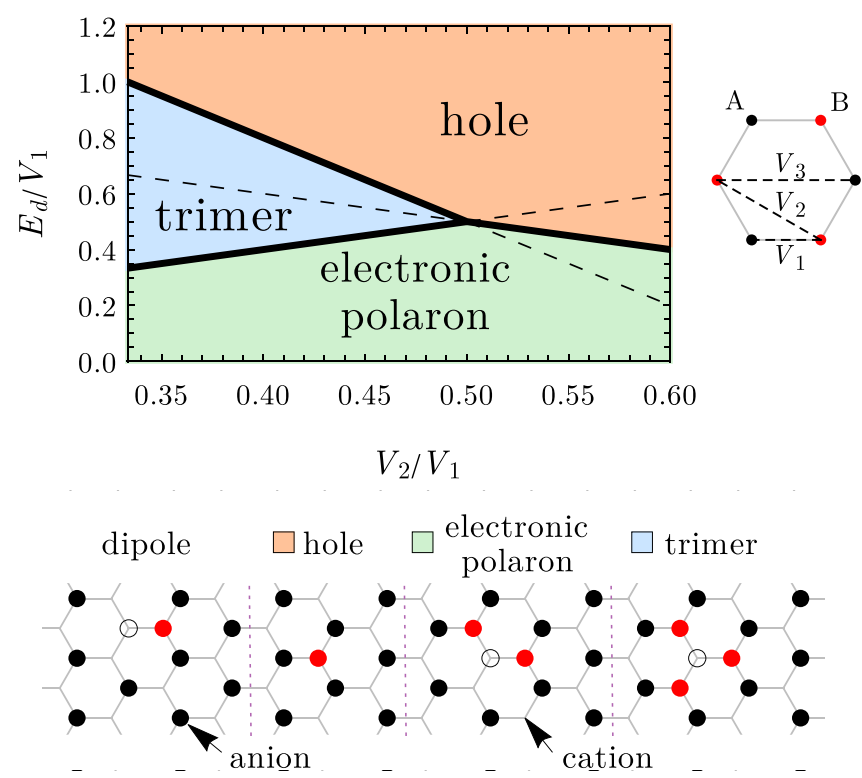

FIG. 1. Phase diagram showing which of the three charged excitations (hole, electronic polaron, or trimer) in the charge-transfer insulator at $n=1$ has the lowest energy per unit charge as a function of the fundamental gap $E_{d}$ [defined in Eq. (3)] and the ratio of nextnearest-neighbor to nearest-neighbor repulsion: $V_{2} / V_{1}$. The dashed lines separate regions where the second lowest energy per charge excitation changes. The bottom panel depicts these excitations where filled circles denote a hole, while an empty circle denotes a hole that is missing from the charge-transfer insulator ground state. Anions and cations are colored black and red, respectively. See Fig. 5 for more information.

and $\mu$ is the chemical potential for holes. Likewise, adding an electron costs energy $E_{-e}+\mu$ with $E_{-e}=-V_{a}$. Transferring a charge from an anion to its adjacent cation creates a charge-transfer exciton, which carries an electric dipole. Its energy cost $E_{d}$ is less than the sum of the electron and the hole energies:

$$
E_{d}=E_{e}+E_{-e}-V_{1}=\Delta+V_{c}-V_{a}-V_{1},
$$

where $V_{1}$ denotes the nearest-neighbor repulsion (see top right of Fig. 1). $E_{d}>0$ defines the fundamental gap of the chargetransfer insulator to local neutral excitations.

Two holes cost energy $2 E_{e}$ when they are spatially separated. Alternatively, consider binding two adjacent holes with a neutral charge-transfer dipole. The result is a charge- $2 e$ composite excitation consisting of three adjacent holes on cations surrounding an electron at the center anion, a "trimer" (see bottom of Fig. 1). Its energy cost, written as $E_{t}=2 E_{e}-$ $\epsilon_{b}$, differs from the two separate holes by a pair binding energy $\epsilon_{b}$ :

$$
\begin{aligned}
\epsilon_{b} & =-E_{d}+2 V_{1}-3 V_{2} \\
& =-\Delta+6 V_{2}^{\prime}-3 V_{2}-3 V_{3}+\cdots \\
& \approx-\Delta+3 V_{2}-3 V_{3}+\cdots,
\end{aligned}
$$

where the second-nearest-neighbor interactions $V_{2}^{\prime}$ and $V_{2}$ are within the $A$ and $B$ sublattice respectively, and ... denotes interactions at larger distance. In the second and third equalities, we have used Eq. (3) and $V_{2}^{\prime} \approx V_{2}$. 
Importantly, in a range of realistic material parameters $\Delta$ and $V_{n}$ (see Fig. 1), $\epsilon_{b}$ is positive so that a charge-2e trimer costs less energy than two individual holes. The energy gain here comes mostly from the simple fact that pairing two holes into a trimer frees up three second-neighbor pairs in the system, which results in the $-3 V_{2}$ energy reduction in Eq. (4). It is remarkable that despite the direct mutual repulsion, two doped holes can, at the expense of energy $\Delta$, tightly bind together with a charge-transfer exciton to lower the total electrostatic repulsion energy.

As an example, for slightly twisted $\mathrm{WSe}_{2} / \mathrm{WS}_{2}$ with a moiré period $L_{\mathrm{M}}=7 \mathrm{~nm}$ and a distance to top and bottom gates equal to $L_{\mathrm{M}}$, a calculation using Wannier functions finds $V_{1}, V_{2}^{\prime}, V_{2}, V_{3}=1.2998,0.4599,0.4780,0.3239$ in units of $e^{2} /\left(\epsilon L_{\mathrm{M}}\right)$, where $\epsilon$ is the permittivity of the dielectric environment [40]. The Coulomb energies at larger separation are much smaller. The trimer binding energy is then found to be $\epsilon_{b}=-14.9+\frac{72.8}{\epsilon} \mathrm{meV}$.

The finding of charge pairing from Coulomb repulsion in a moiré superlattice is our first main result, which forms the basis for our theory at finite doping. Notably, previous works found an effective attraction between two added charges in small Hubbard-model clusters at intermediate $U / t$ [42-44] or with extended interactions [45]. In this study of extended moiré systems, the pair binding energy is already manifest in the strong-coupling limit $t_{i j}=0$ without explicitly invoking any charge fluctuation or weakly coupled clusters. Note, however, the fact crucial to our analysis that doped charges occupy quantized orbitals localized around discrete lattice points, rather than taking arbitrary positions in the continuum. It is this quantum-mechanical effect that leads to the quantized energy of trimers.

We also mention in passing that, besides holes and trimers, other composite excitations can be energetically favorable in certain parameter ranges. These include (1) the electronic polaron $(q=e)$, which is a bound state of a hole and a dipole, and (2) higher-charge excitations with $q \geqslant 3 e$. By comparing the energy cost of different types of charged excitations at filling $n=1$, we identify the excitation with the least energy per unit charge - the cheapest charge excitation (see Fig. 1).

\section{PAIR DENSITY WAVES}

Building on these results on few-body excitations, we now study the many-body physics of TMD heterobilayers at fillings $n=1+\delta$ with small $\delta>0$, which are doped chargetransfer insulators. In particular, we develop an analytically controlled theory to predict pair density waves and superconductivity under appropriate conditions.

For large moiré periods where the Coulomb interaction dominates the kinetic energy, a periodic array of charge excitations of the least cost is favored. If the cheapest excitation is a charge- $e$ hole, then the ground state of $H_{0}$ will be a commensurate charge density wave at, e.g., the dopings $\delta=1 / 7$, 1/4, or 1/3, shown in Figs. 2(a), 2(c), and 2(e). Similar charge density waves have been discussed in the context of twisted bilayer graphene [46] and extended Hubbard models [47-49].

On the other hand, if the cheapest charge excitation is a charge- $2 e$ trimer, then the ground state of $H_{0}$ is a pair density wave [50] with a commensurate periodicity at dopings such

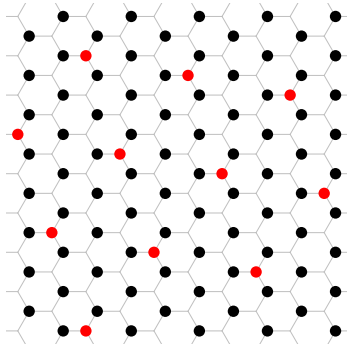

(a) $\delta=1 / 7 \approx 0.143$ $q=1, r_{1}=2, r_{2}=1$

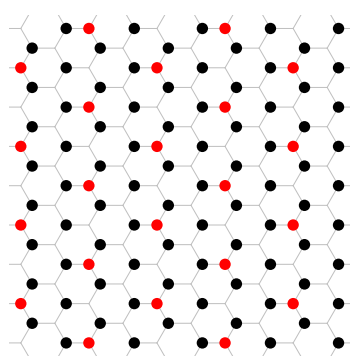

(c) $\delta=1 / 4=0.25$

$q=1, r_{1}=2, r_{2}=0$

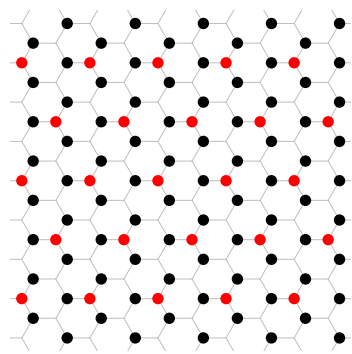

(e) $\delta=1 / 3 \approx 0.333$

$q=1, r_{1}=1, r_{2}=1$

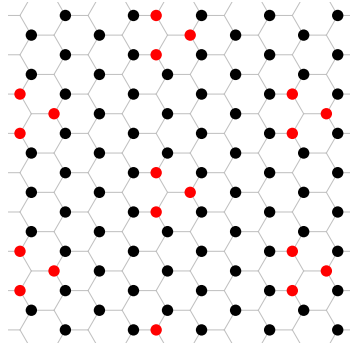

(b) $\delta=1 / 8=0.125$ $q=2, r_{1}=4, r_{2}=0$

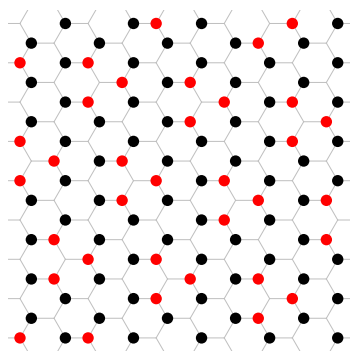

(d) $\delta=2 / 7 \approx 0.286$ $q=2, r_{1}=2, r_{2}=1$

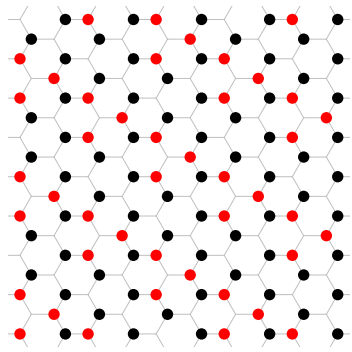

(f) $\delta=1 / 3 \approx 0.333$
FIG. 2. Examples of (a, c, e) commensurate charge and (b, d, f) pair density waves at various fractional fillings $n=1+\delta$. The charge $q$ and integers $r_{1}$ and $r_{2}$ from Eq. (5) are listed for the triangular Wigner crystals of holes $(q=e)$ and of trimers $(q=2 e)$.

as $\delta=1 / 8,2 / 7$, or $1 / 3$, shown in Figs. 2(b), 2(d), and 2(f). In particular, the pair density wave at $\delta=2 / 7$ [shown in Fig. 2(d)] can be viewed as the closest packing of trimers with negligible intertrimer interaction (involving only $V_{n \geqslant 5}$ ), in contrast to Fig. 2(f) (which involves $V_{n \geqslant 2}$ ). Possible evidence of such a state can be seen from the Berkeley group's recent data (see Fig. 7). Furthermore, possible evidence for one of the $\delta=1 / 3$ states was observed in Ref. [29].

More generally, we predict that at low temperature, clean TMD heterobilayers with a large moiré period should exhibit a sequence of insulating density wave states at the following fillings when a commensurate triangular lattice of charge excitations is formed:

$$
n=1+\frac{q}{r_{1}^{2}+r_{1} r_{2}+r_{2}^{2}} \quad\langle\quad
$$

The integer $q>0$ is the charge per excitation, and the integers $r_{i} \geqslant 0$ specify the Bravais lattice vector $r_{1} \mathbf{b}_{1}+r_{2} \mathbf{b}_{2}$ of the 


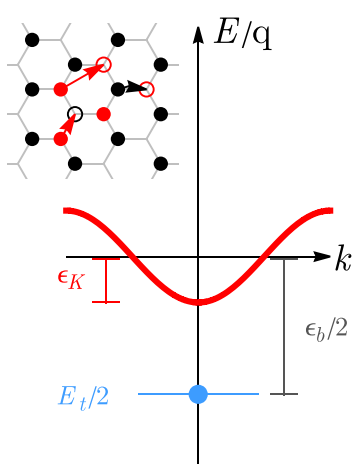

(a) pair density wave or $\mathrm{BEC}$

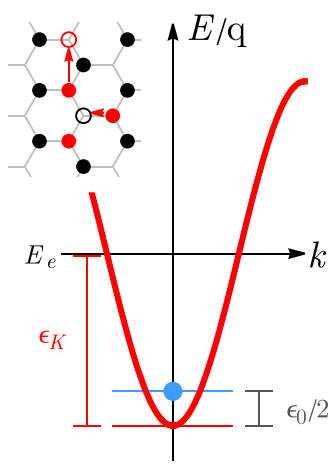

(b) resonantly-paired superconductivity
FIG. 3. Energy spectrum of the dispersive hole band and the trimer. (a) When the trimer energy per charge, $E_{t} / 2$, lies below the hole band bottom, a pair density wave (PDW) or Bose-Einstein condensate of trimers is expected. Inset: A third-order trimer hopping process. (b) When $E_{t} / 2$ lies close to the hole band bottom, low-energy holes interact resonantly with trimers, leading to superconductivity. Inset: A second-order process in which a trimer decays into two holes separated by a distance $2 L_{\mathrm{M}}$.

commensurate density wave, with $\mathbf{b}_{1}, \mathbf{b}_{2}$ denoting the two lattice vectors of the moiré honeycomb lattice shown in Eq. (5).

\section{RESONANTLY PAIRED SUPERCONDUCTIVITY}

While Coulomb repulsion favors density waves, the singleparticle hopping term $H_{K}$ favors charge delocalization. In the following, we study the competition between Coulomb energy and kinetic energy in the interesting and experimentally relevant parameter regime $\epsilon_{b}>0$, where the trimer has a lower energy than two separated holes in the limit $t_{i j}=0$ (the trimer region of Fig. 1). For simplicity, we consider the scenario in which the system is fully spin polarized, which is experimentally realized in $\mathrm{WSe}_{2} / \mathrm{WS}_{2}$ under a small magnetic field (less than $1 \mathrm{~T}$ at $1.6 \mathrm{~K}$ ) [27]. Note that an applied magnetic field will not weaken charge pairing since charge pairing occurs through electrostatic interactions and without double occupancy.

Single-particle hopping $t_{i j}$ between cation sites on the triangular sublattice leads to a dispersive band of doped holes. Then, the lowest-energy hole excitation is in the delocalized state at the bottom of this band. Its energy is $E_{e}-\epsilon_{K}$, where $\epsilon_{K}>0$ is proportional to the hopping integral $t$. In contrast, as a composite excitation, the trimer can only hop via a highorder process involving high-energy intermediate states [see Fig. 3(a)]. In the strong-coupling regime, the trimer hopping integral is on the order of $t^{3} / V^{2}$ with $V \sim e^{2} / \epsilon L_{\mathrm{M}}$ and thus likely negligible.

With $t_{i j} \neq 0$, it is important to consider the hybridization between trimers and holes. This occurs when a constituent $e$ charge in a trimer hops back to the center anion, leaving behind two adjacent holes in a high-energy state due to their strong mutual repulsion $V_{2}$. To lower their energy, these two remaining holes tend to hop away from each other. Thus, converting a trimer into two weakly interacting holes that are sufficiently apart requires at least a second-order process, shown in Fig. 3(b). Due to the large energy barrier in the pathway between the trimer and two free holes, the trimer remains a long-lived quasibound state.

Therefore, at finite doping, we are faced with a mixture of charge- $e$ holes and charge- $2 e$ trimers that are hybridized and at a total charge density $\delta$. For small doping $\delta$, the typical distance between doped charges is much larger than the moiré period $L_{\mathrm{M}}$; hence the underlying moiré lattice only plays a minor role. Thus, the essential low-energy physics is captured by a boson-fermion model in the continuum, which we introduce for doped charge-transfer insulators:

$$
\begin{aligned}
H_{\delta} & =\int d \mathbf{r} \sum_{\sigma= \pm} \psi_{\sigma}^{\dagger}\left(-\frac{\nabla^{2}}{2 m}\right) \psi_{\sigma}+\epsilon_{0} \phi^{\dagger} \phi+g\left(\phi \psi_{+}^{\dagger} \psi_{-}^{\dagger}+\phi^{\dagger} \psi_{-} \psi_{+}\right)-\mu n(\mathbf{r})+\frac{1}{2} \int d \mathbf{r}^{\prime} V\left(\left|\mathbf{r}-\mathbf{r}^{\prime}\right|\right) n(\mathbf{r}) n\left(\mathbf{r}^{\prime}\right) \\
\text { with } n(\mathbf{r}) & =\sum_{\sigma= \pm} \psi_{\sigma}^{\dagger} \psi_{\sigma}+2 \phi^{\dagger} \phi,
\end{aligned}
$$

where $\psi$ and $\phi$ denote the itinerant hole and immobile trimer, respectively.

According to band-structure calculations for $\mathrm{WSe}_{2} / \mathrm{WS}_{2}$, $t_{i j}>0$ between nearest-neighbor cations. Hence the band of doped holes has two degenerate minima at corners of the moiré Brillouin zone $\pm \mathbf{K}=\left(0, \pm \frac{4 \pi}{3 L_{\mathrm{M}}}\right)$ [30], which are denoted by the valley index $\sigma= \pm$ in Eq. (6). $m \propto 1 / t$ is the effective mass at the band bottom. $\epsilon_{0}=-\epsilon_{b}+2 \epsilon_{K}$ with $\epsilon_{K} \propto$ $t$ is the energy difference between a trimer and two delocalized holes at the band bottom. Since the hopping integral $t$ changes significantly with the moiré period, the detuning parameter $\epsilon_{0}$ is tunable by varying the twist angle.

Two types of interactions are included in our model Hamiltonian $H_{\delta}$ and play dominant roles in the low-density regime $\delta \ll 1$ : (1) the extended Coulomb interaction $V(r)$, whose range is determined by the distance to metallic gates, and (2) the local hybridization $g$ between holes and trimers. The form of the hybridization is dictated by symmetry. The trimer state with maximal spin is invariant under threefold rotation around the center anion and odd under reflection which exchanges a pair of holes (fermions). Therefore, the trimer hybridizes with a valley-singlet pair of holes $\psi_{+}^{\dagger} \psi_{-}^{\dagger}$, which transforms in the same way (note that reflection interchanges $\pm \mathbf{K}$ ). Despite being a weak local interaction, the hole-trimer hybridization can have dramatic consequences for our system at low density.

Our model exhibits an enormously rich phase diagram resulting from the interplay of (1) the kinetic energy of holes, (2) the binding energy of trimers, (3) Coulomb repulsion between charges, and (4) the hybridization between holes and trimers. In particular, we show that pair density wave and 
superconducting ground states emerge in certain parameter regimes.

If the hopping integral $t$ in the microscopic Hamiltonian (1) is small compared to the binding energy of trimers $\epsilon_{b}>0$ [see Fig. 3(a)], then the kinetic energy of holes is of minor importance. Therefore, $\epsilon_{0} \approx-\epsilon_{b}<0$, and doped charges go into trimers. At finite charge density up to $\delta=1 / 3$, the (screened) Coulomb repulsion $V(r)$ between charges leads to a Wigner crystal of charge- $2 e$ trimers, which takes a triangular lattice structure. At the dopings specified by Eq. (5), this trimer Wigner crystal is commensurate with and pinned by the moire potential. The resulting state is a gapped and insulating pair density wave. At sufficiently low doping where the average distance between trimers exceeds the range of $V(r)$, the density wave state becomes fragile and potentially unstable to Bose condensation of trimers when their small hopping amplitudes are taken into account.

As the hopping integral $t$ increases, the bottom of the hole band is lowered and eventually falls below $E_{t} / 2$, as shown in Fig. 3(b). Correspondingly, the bare detuning parameter $\epsilon_{0}$ changes from negative to positive. The true detuning parameter $\epsilon$ is renormalized by the hole-trimer hybridization: $\epsilon=\epsilon_{0}-o\left(g^{2}\right)$ [51]. At negative detuning $\epsilon<0$, there exists a true bound state of two $e$ charges, which is a superposition of a trimer and a cloud of two holes. At positive detuning $\epsilon>0$, no such bound state exists. However, when the detuning is small, low-energy holes and trimers are strongly hybridized. Two $e$ charges at low energy or large de Broglie wavelength scatter resonantly in the $s$-wave valley-singlet channel. Such resonant interaction is universally characterized by a large scattering length, which can exceed the range of screened Coulomb repulsion $V(r)$. The scattering length is positive (negative) for $\epsilon>0(\epsilon<0)$ and diverges at $\epsilon=0$.

The physics of the resonant interaction via the trimer channel is reminiscent of a Feshbach resonance in an ultracold Fermi gas [38]. Under the resonant condition, the ground state of our system at low doping is a superconductor with same-spin pairing in the valley-singlet channel (corresponding to $f$-wave pairing symmetry on the honeycomb lattice). A crossover between the Bose-Einstein condensate (BEC) and Bardeen-Cooper-Schrieffer (BCS) regimes [39] can be achieved as a function of doping density $\delta$ and the detuning $\epsilon$. See Appendix A for a mean-field theory analysis and more microscopic details.

It should be noted that the origin of the resonant interaction in our system is completely different from the case of cold atoms. Unlike the molecule formed by two atoms in empty space, the trimer is a charge-transfer excitation in a many-body "vacuum" at the filling $n=1$. It is remarkable that spin-polarized superconductivity can be realized in a solidstate system with purely Coulomb repulsion. We emphasize that our prediction of superconductivity is rigorous in the regime of weak kinetic energy $(t \ll V)$, polarized spins, small doping $(\delta \ll 1)$, and resonance (small $\left.\epsilon_{0}\right)$. These limits can be reasonably achieved in moiré TMD systems. It will be interesting to consider relaxing some of these assumptions (e.g., polarized spins) in future work since other possibilities are likely outside of this regime.

Note that these limits are not as applicable to cuprates, which are antiferromagnetic at zero doping and become superconducting only under a sufficient doping after antiferromagnetism is destroyed.

\section{DISCUSSION}

To summarize, we developed a strong-coupling theory to predict electron pairing from repulsion via charge-transfer excitations in TMD heterobilayers. We further predict insulating pair density wave states at a sequence of doping levels. Finally, we show that with the increase of electron itinerancy, the resonant interaction between itinerant holes and local charge$2 e$ pairs leads to unconventional superconductivity. Since our theory is based on a general doped charge-transfer insulator, our theory is broadly applicable to other charge-transfer insulating moiré materials, such as $\mathrm{MoS}_{2} / \mathrm{MoS}_{2}$ and other TMD homobilayers in an electric field [26,52,53]. Chargetransfer physics occurs when $U>\Delta$, where $U$ is large due to localized orbitals in moiré TMD bilayers [54] and $\Delta$ can be arbitrarily small in TMD homobilayers where it is proportional to the applied electric field. Our pairing mechanism may also shed light on other moiré materials, such as twisted graphene multilayers, where charge redistribution under doping may be important $[55,56]$ and spin-polarized superconductivity may have been observed $[57,58]$. We hope our prediction of fascinating correlated states in moiré materials can stimulate further activity and find experimental realization soon.

\section{ACKNOWLEDGMENTS}

We are grateful to Steve Kivelson for a stimulating and insightful discussion. We thank Jason Alicea, Chenhao Jin, Patrick Lee, Stevan Nadj-Perge, Gil Refael, Alex Thomson, and Sagar Vijay for helpful conversations. We thank Yang Zhang, Noah Yuan, and especially Tongtong Liu for collaboration on related works, and Kin Fai Mak, Jie Shan, and Feng Wang for valuable discussions on experiments. It is our pleasure to thank the organizers of the KITP conference "Topological Quantum Matter: From Fantasy to Reality," where this work was initiated. K.S. is supported by the Walter Burke Institute for Theoretical Physics at Caltech. L.F. is supported by the DOE Office of Basic Energy Sciences under Award No. DE-SC0018945, and in part by a Simons Investigator award from the Simons Foundation.

\section{APPENDIX A: ADDITIONAL DETAILS}

In this Appendix, we provide extra details on the lowenergy excitations, followed by a mean-field theory analysis of the superconducting states starting from the lattice model in Eq. (1). We consider the low-density and strongly interacting limit

$$
n-1=\delta \ll 1 \quad \text { and } \quad t_{i j} \ll V_{2} \text {, }
$$

where $V_{n}$ is the Coulomb repulsion between $n$ th-nearest neighbors (see Fig. 4). We also assume that the spins are fully polarized, e.g., by an external magnetic field. 


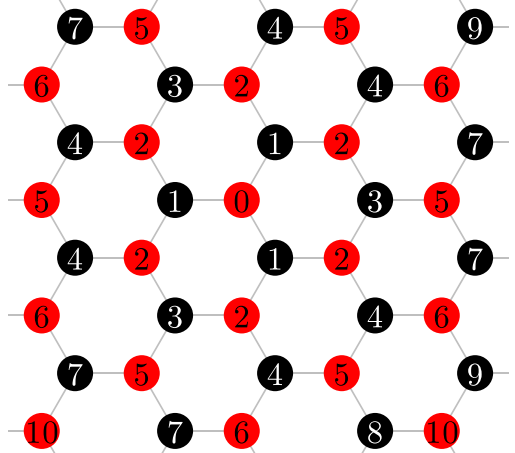

FIG. 4. $n$ th-nearest neighbors on a honeycomb lattice. Throughout this work, $V_{n}$ denotes the Coulomb repulsion between $n$ th-nearest neighbors. We sometimes differentiate between $V_{n}^{A A}$ and $V_{n}^{B B}$ for repulsions between a pair of sites on the $A$ or $B$ sublattice. $n$ th-nearest neighbors are separated by $a_{n}$ with $a_{n=1,2,3,4,5} / a_{1}=1, \sqrt{3}, 2, \sqrt{7}, 3$. $L_{\mathrm{M}}=a_{2}$ is the moiré period.

\section{Electrostatics}

In Fig. 1 of the main text, we showed which of the following three excitations has the smallest energy per charge, $E / q$ :

hole $(q=1): \quad E_{-e}$,

trimer $(q=2): \quad E_{t}=2 E_{e}+E_{d}-2 V_{1}+3 V_{2}^{B B}$,

electronic polaron $(q=1): \quad E_{e p}=E_{e}+E_{d}-V_{1}+V_{2}^{B B}$.

Remarkably, for the above three excitations, this only requires knowing $E_{d} / V_{1}$ and $V_{2}^{B B} / V_{1} ; \Delta$ and all $V_{n}$ are effectively absorbed into these two ratios.

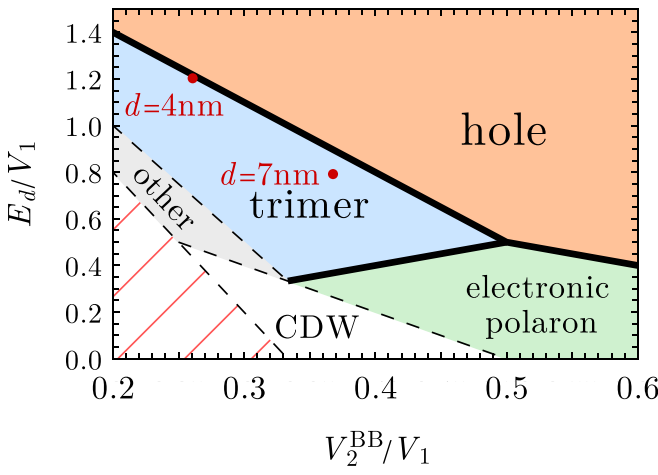

(a)

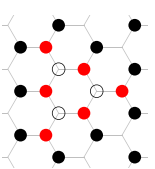

(b)
FIG. 5. (a) A detailed excitation phase diagram of the lowest energy per charge $(E / q)$ excitation. The red dots mark estimates for a slightly twisted $\mathrm{WSe}_{2} / \mathrm{WS}_{2}$ inside a dielectric environment with permittivity $\epsilon=3$ and for different distances $d$ to metallic gates. In the "other" region, other excitations have the least $E / q$, such as the charge- $3 e$ excitation shown in (b). The bottom left region (white with red stripes) is inaccessible (when $V_{n \geqslant 3}=0$ ) as it would require $\Delta<0$. The dashed lines are drawn for $V_{n \geqslant 3}=0$. See Appendix A 1 for more details.

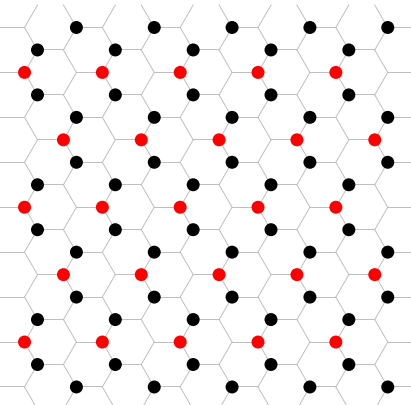

(a)

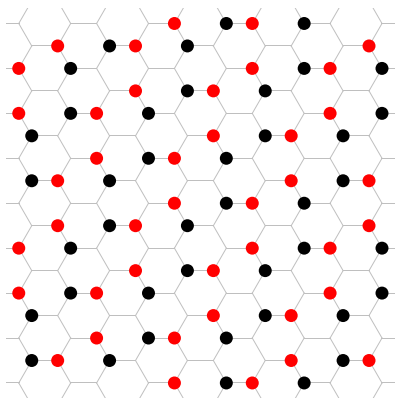

(b)
FIG. 6. Possible instabilities of the charge-transfer insulator. (a) A Wigner crystal of dipoles, which is a possible state when the dipole energy is negative, $E_{d}<0$. (b) The charge density wave that occurs in the CDW region (white) of Fig. 5 when $V_{n \geqslant 4}=0$ and $V_{3} \ll V_{2}$.

It is remarkable that charge- $2 e$ pairing can occur from just repulsive interactions. The nontrivial charge-transfer insulating background is essential for the trimer stability; a trimer is not stable in the vacuum [59]. As an aid to intuition, we give an even simpler example of how this can occur in a finite system in Appendix B.

In Fig. 5, we show a more detailed diagram of the smallest $E / q$ excitation when arbitrary excitations are considered We also check for instabilities (shown in Fig. 6) of the charge transfer insulator, which occur in the "CDW" region of Fig. 5 and when $E_{d}<0$. The energy of other excitations and these instabilities depend on more than just $E_{d} / V_{1}$ and $V_{2}^{B B} / V_{1}$. Therefore, we show the locations of these other excitations and instabilities for the simple case when $V_{n \geqslant 3}=0$. In Fig. 5, dashed lines are used to depict boundaries that assume $V_{n \geqslant 3}=0$.

We also estimate where in the phase diagram a slightly twisted $\mathrm{WSe}_{2} / \mathrm{WS}_{2}$ with a moiré period $L_{\mathrm{M}}=7 \mathrm{~nm}$ could be, which we show in Fig. 5 using red dots. The locations are calculated using $\Delta=14.9 \mathrm{meV}$ and the values of $V_{n}$ are shown in Table I. $\Delta$ and $V_{n}$ were calculated using Wannier orbitals and a Coulomb interaction $V(r)$ that is screened by a pair of metallic gates, each a distance $\pm d$ from the TMD heterobilayer [40]. By modeling the gates as perfect conductors, the screened Coulomb interaction can be calculated using the

TABLE I. The values of $V_{n}$ (in units of $\frac{e^{2}}{\epsilon L_{M}}=\frac{205.7}{\epsilon} \mathrm{meV}$ with $L_{\mathrm{M}}=7 \mathrm{~nm}$ ) used to estimate the location (denoted by red dots in Fig. 5) of slightly twisted $\mathrm{WSe}_{2} / \mathrm{WS}_{2}$ in the excitation phase diagram: (a) $d=4 \mathrm{~mm}$, (b) $d=7 \mathrm{~mm}$.

\begin{tabular}{lcccc}
\hline \hline$n$ & 0 & 1 & 2 & 3 \\
\hline & & (a) $d=4 \mathrm{~nm}$ & & \\
$V_{n}^{A A}$ & 3.7769 & 0.9479 & 0.2292 & \\
$V_{n}^{A B}$ & & & 0.2472 & \\
$V_{n}^{B B}$ & 2.9828 & (b) $d=7 \mathrm{~mm}$ & & \\
$V_{n}^{A A}$ & 4.2407 & & 0.4599 & \\
$V_{n}^{A B}$ & & 1.2998 & 0.3239 \\
$V_{n}^{B B}$ & 3.4132 & & 0.4780 & \\
\hline \hline
\end{tabular}




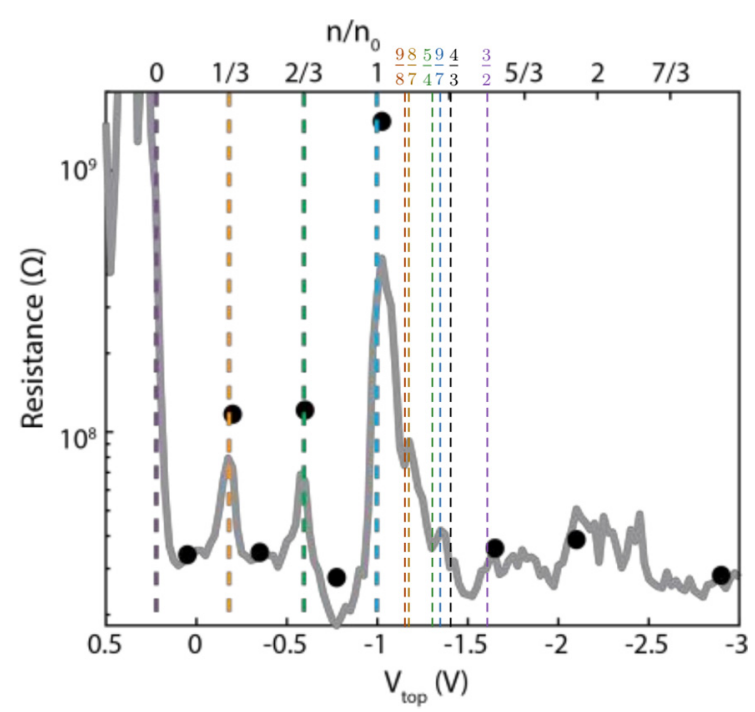

FIG. 7. Resistance of the Berkeley group's $\mathrm{WSe}_{2} / \mathrm{WS}_{2}$ moiré device as a function of gate voltage, which determines the electron filling, $n$. The figure is copied from Ref. [28]. We add vertical lines to indicate various Wigner crystal fillings. The blue line at filling $n=9 / 7$ is particularly interesting because it shows a large resistance peak at the same filling as the pair density wave in Fig. 2(d). Energetically favorable charge density waves at filling $n=3 / 2$ are shown in Fig. 8.

method of image charges, yielding [60]

$$
V(r)=\frac{e^{2}}{\epsilon} \sum_{z \in \mathbb{Z}} \frac{(-1)^{z}}{\sqrt{r^{2}+(2 d z)^{2}}} .
$$

When $r \gg d, V(r)$ decays exponentially.

In Fig. 7, we point out possible experimental evidence of insulating pair density waves of trimers from recently observed resistivity peaks [28]. See also Ref. [29] for evidence for additional charge orders.

\section{Mean-field theory of superconductivity}

Here, we study the mean-field theory of trimer superconductivity. Suppose that we are near the edge of the trimer region of the phase diagram, so that the trimer binding energy, $\epsilon_{b}$ [Eq. (4)], is small:

$$
0<\epsilon_{b} \ll V_{2} .
$$

We also assume that excitations other than the charge- $e$ hole and charge- $2 e$ trimers (such as the dipole and electronic polaron) have a large energy cost $\sim V_{2}$ so that they can be ignored.

The low-energy Hamiltonian thus consists of only the mobile holes $c_{\mathbf{k}}$ on the cations, and bosonic trimers $b_{a}$ centered on the anions:

$$
\begin{aligned}
H_{\text {eff }}= & \sum_{\mathbf{k}} \epsilon(\mathbf{k}) c_{\mathbf{k}}^{\dagger} c_{\mathbf{k}}+\left(\epsilon_{0}-2 \mu\right) \sum_{a} b_{a}^{\dagger} b_{a} \\
& -\frac{\tilde{g}\left(\mathbf{k}-\mathbf{k}^{\prime}\right)}{2 \sqrt{N}} \sum_{\mathbf{k}, \mathbf{k}^{\prime}}\left(b_{\mathbf{k}+\mathbf{k}^{\prime}}^{\dagger} c_{\mathbf{k}} c_{\mathbf{k}^{\prime}}+\text { H.c. }\right)+\cdots,
\end{aligned}
$$

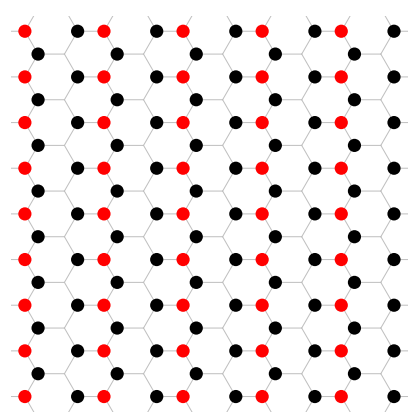

(a) $\delta=1 / 2$

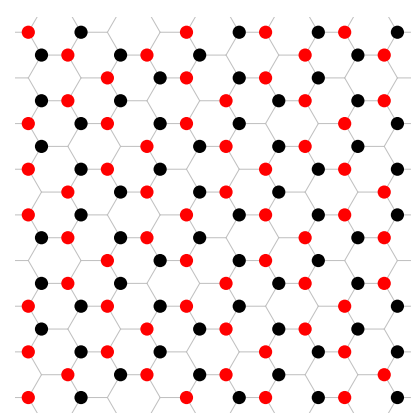

(b) $\delta=1 / 2$
FIG. 8. A charge density wave state at doping $\delta=1 / 2$ that is typical of the (a) hole and (b) trimer regimes of the excitation phase diagram in Fig. 5.

where $b_{\mathbf{k}}^{\dagger}=N^{-1 / 2} \sum_{a} e^{i \mathbf{k} \cdot a} b_{a}^{\dagger}$ and $N$ is the number of anion sites.

The first term gives the dispersion of the holes, which hop on the triangular lattice of cations. At low density $(\delta \ll 1), \epsilon(\mathbf{k})$ can be expanded about its band minima $\pm \mathbf{K}=$ $\left(0, \pm \frac{4 \pi}{3 L_{\mathrm{M}}}\right)$ :

$$
\epsilon(\mathbf{k} \pm \mathbf{K})=\frac{1}{2 m} k^{2}-\mu+O\left(k^{3}\right),
$$

$m \approx 2 L_{\mathrm{M}}^{2} / 27 t_{2}$, where $t_{n}$ is the hopping energy between $n$ thnearest neighbors. We have shifted $\epsilon(\mathbf{k})$ so that $\epsilon( \pm \mathbf{K})=0$.

The second term sets the energy cost of trimers. $b_{a}^{\dagger}$ creates a trimer centered at the anion $a$, and $b_{\mathbf{k}}^{\dagger}$ creates a trimer with momentum k. $\epsilon_{0} \approx 6 t_{2}-\epsilon_{b}$ is the energy difference between a trimer and two holes at the band minima $\pm \mathbf{K}$.

The last term accounts for the conversion between a trimer and a pair of holes. The triangular symmetry of the cation sublattice constrains the conversion amplitude to the following form: $\tilde{g}(\mathbf{q})=\tilde{g} \sum_{i=1}^{3} \frac{2}{3 \sqrt{3}} \sin \left(\mathbf{q} \cdot \mathbf{r}_{i}\right)+$ $\ldots$, where "..." denotes higher-order moments and $\mathbf{r}_{i}=$ $\sqrt{3} \sin \left(\frac{2 \pi}{3} i\right) \hat{\mathbf{x}}-\sqrt{3} \cos \left(\frac{2 \pi}{3} i\right) \hat{\mathbf{y}}$ are second-nearest-neighbor displacements. The normalization is such that $\tilde{g}(\mathbf{K})=$ $-\tilde{g}(-\mathbf{K})=\tilde{g}(-2 \mathbf{K})=\tilde{g}$. $\tilde{g}$ can be calculated perturbatively; the leading contribution comes from the process shown in Fig. 3(b) and has amplitude $\tilde{g} \sim t_{1} t_{2} / V_{2}$ [61].

Expanding $H_{\text {eff }}$ about the band minima $\pm \mathbf{K}$ leads to

$$
\begin{aligned}
H_{\mathrm{eff}} \approx & \sum_{\mathbf{k}, \pm} \epsilon_{k} c_{\mathbf{k}, \pm}^{\dagger} c_{\mathbf{k}, \pm}+\left(\epsilon_{0}-2 \mu\right) \sum_{a} b_{a}^{\dagger} b_{a} \\
& -\frac{\tilde{g}}{\sqrt{N}} \sum_{\mathbf{k}, \mathbf{k}^{\prime}}\left(b_{\mathbf{k}+\mathbf{k}^{\prime}}^{\dagger} c_{\mathbf{k},-} c_{\mathbf{k}^{\prime},+}+\text { H.c. }\right)+\cdots,
\end{aligned}
$$

where $c_{\mathbf{k}, \pm} \sim c_{\mathbf{k} \pm \mathbf{K}}$ denotes the new fermion operators expanded about $\pm \mathbf{K}$ and $\epsilon_{k}=\frac{1}{2 m} k^{2}-\mu$ is the dispersion.

The "..." in $H_{\text {eff }}$ denotes other terms that could be included in $H_{\text {eff }}$. We ignore these terms in the following meanfield analysis because we do not expect these terms to be relevant in the resonantly paired superconductivity regime of interest. To justify this, consider two potentially important kinds of terms that we are omitting. The first is a trimer kinetic energy term, $-t_{t} \sum_{a^{\prime} a} b_{a^{\prime}}^{\dagger} b_{a}$, where $t_{t} \sim t^{3} / V^{2}$ is the trimer hopping energy, resulting from the perturbative process shown in Fig. 3(a). However, we expect that near resonance, this term 
is negligible compared to the effective boson mass resulting from the coupling $\tilde{g}$ to the fermions. The second potentially important terms are 4-fermion interactions, such as $V_{i j} n_{i} n_{j}$. However, we soon see [from Eq. (A17)] that near resonance, the fermion and boson operators scale as $c \sim b \sim O(\sqrt{\delta})$ at low density $\delta$. Therefore, the first term in $H_{\text {eff }}$ is $O(t \delta)$; the third term contributes $O\left(\delta^{3 / 2} \tilde{g}\right) \sim O\left(\delta^{3 / 2} t^{2} / V_{2}\right)$; and a 4-fermion interaction would contribute $O\left(\delta^{2} V\right)$. Thus, we expect that the 4-fermion interaction is negligible when $\delta^{2} V \ll$ $\delta^{3 / 2} t^{2} / V$, i.e., at the sufficiently low doping $\delta \ll(t / V)^{4}$.

To make a connection to $H_{\delta}$ in Eq. (6) of the main text, note that $\psi^{\dagger} \sim L_{\mathrm{M}}^{-1} c^{\dagger}$ and $\phi^{\dagger} \sim L_{\mathrm{M}}^{-1} b^{\dagger}$. Then $g$ in $H_{\delta}$ and $\tilde{g}$ in $H_{\text {eff }}$ [Eq. (A7)] are related by $g \sim L_{\mathrm{M}} \tilde{g}$. In the following, we use lattice units where the distance between nearest-neighbor sites is 1 , so that the distance between next-nearest neighbors is $L_{\mathrm{M}}=\sqrt{3}$.

To make analytical progress, we consider the following mean-field approximation:

$$
\begin{aligned}
b_{a}^{\dagger} b_{a}= & b_{a}^{\dagger}\left\langle b_{a}\right\rangle+\left\langle b_{a}^{\dagger}\right\rangle b_{a}-\left\langle b_{a}^{\dagger}\right\rangle\left\langle b_{a}\right\rangle \\
& +\left(b_{a}^{\dagger}-\left\langle b_{a}^{\dagger}\right\rangle\right)\left(b_{a}-\left\langle b_{a}\right\rangle\right) \\
\approx & b_{a}^{\dagger}\left\langle b_{a}\right\rangle+\left\langle b_{a}^{\dagger}\right\rangle b_{a}-\left\langle b_{a}^{\dagger}\right\rangle\left\langle b_{a}\right\rangle, \\
b_{\mathbf{k}+\mathbf{k}^{\prime}}^{\dagger}, c_{\mathbf{k},-} c_{\mathbf{k}^{\prime},+} & \approx\left\langle b_{\mathbf{k}+\mathbf{k}^{\prime}}^{\dagger}\right\rangle c_{\mathbf{k},-} c_{\mathbf{k}^{\prime},+} .
\end{aligned}
$$

With this approximation, the low-energy Hamiltonian becomes quadratic:

$$
\begin{aligned}
H_{\mathrm{MF}}= & \sum_{\mathbf{k}}\left(\begin{array}{l}
c_{+\mathbf{k},+} \\
c_{-\mathbf{k},-}^{\dagger}
\end{array}\right)^{\dagger}\left(\begin{array}{cc}
+\epsilon_{k} & -\Delta_{b} \\
-\Delta_{b} & -\epsilon_{k}
\end{array}\right)\left(\begin{array}{l}
c_{+\mathbf{k},+} \\
c_{-\mathbf{k},-}^{\dagger}
\end{array}\right) \\
& +\frac{\epsilon_{0}-2 \mu}{\tilde{g}^{2}} \Delta_{b}^{2}, \\
\Delta_{b}= & \tilde{g}\left\langle b_{a}\right\rangle .
\end{aligned}
$$

$\Delta_{b}$ is the superconducting order parameter. $\Delta_{b}>0$ is assumed to be positive (without loss of generality).

The ground-state energy density is

$$
\begin{gathered}
\frac{E_{\mathrm{MF}}}{N}=-\int_{E} D(E) \sqrt{E^{2}+\Delta_{b}^{2}}+\frac{\epsilon_{0}-2 \mu}{\tilde{g}^{2}} \Delta_{b}^{2}, \\
D(E)=\int_{\mathbf{k}} \delta\left(E-\epsilon_{k}\right)= \begin{cases}2 \pi m & -\mu<E<W \\
0 & \text { otherwise, }\end{cases}
\end{gathered}
$$

where $D(E)$ is the density of single-particle states, and $\int_{\mathbf{k}}=$ $\int \frac{d^{3} \mathbf{k}}{(2 \pi)^{2}} \Theta\left(W-\epsilon_{k}\right)$ integrates over momentum states with energy $\epsilon_{k}<W$. W is a UV cutoff which can be taken to be $W=(2 \pi m)^{-1}-\mu \approx(2 \pi m)^{-1}$ so that $\int_{E} D(E)=1$; this is roughly equal to the bandwidth $9 t_{2} \approx 2 m^{-1}$. Evaluating the integral yields

$$
\begin{aligned}
\frac{E_{\mathrm{MF}}}{N}= & -\pi m\left[W \sqrt{W^{2}+\Delta_{b}^{2}}+\Delta_{b}^{2} \log \left(W+\sqrt{W^{2}+\Delta_{b}^{2}}\right)\right. \\
& +\mu \sqrt{\mu^{2}+\Delta_{b}^{2}}+\Delta_{b}^{2} \log \left(\mu+\sqrt{\mu^{2}+\Delta_{b}^{2}}\right) \\
& \left.-2 \Delta_{b}^{2} \log \Delta_{b}\right]+\frac{\epsilon_{0}-2 \mu}{\tilde{g}^{2}} \Delta_{b}^{2} .
\end{aligned}
$$

The superconducting order parameter $\Delta_{b}$ can be calculated by minimizing the energy as a function of $\Delta_{b}$, which yields

$$
\Delta_{b}=\frac{\sqrt{W^{2}+\mu^{2}+2 W \mu \cosh \frac{\epsilon_{0}-2 \mu}{\pi m \tilde{g}^{2}}}}{\sinh \frac{\epsilon_{0}-2 \mu}{\pi m \tilde{g}^{2}}} .
$$

$\Delta_{b}$ depends strongly on the chemical potential $\mu$, which can be obtained from the filling constraint:

$$
\begin{aligned}
\delta & =\delta_{c}+2 \delta_{b}, \quad \delta_{c}=\left\langle c_{i}^{\dagger} c_{i}\right\rangle=4 \pi m \mu, \\
\delta_{b} & =\left\langle b_{a}^{\dagger} b_{a}\right\rangle \approx \frac{\Delta_{b}^{2}}{\tilde{g}^{2}},
\end{aligned}
$$

where $\delta_{c}$ is the density of holes. $\delta_{b}$ is the density of bosonic trimers, which we approximate at the mean-field level: $\left\langle b_{a}^{\dagger} b_{a}\right\rangle \approx\left\langle b_{a}^{\dagger}\right\rangle\left\langle b_{a}\right\rangle=\Delta_{b}^{2} / \tilde{g}^{2}$.

There are two regimes: (1) BCS superconductivity when $\frac{\epsilon_{0}-2 \mu}{\pi m \tilde{g}^{2}} \gg 1$, and (2) resonantly paired superconductivity when $\epsilon_{0} \approx 2 \mu$.

\section{a. BCS superconductivity regime} as

When $\frac{\epsilon_{0}-2 \mu}{\pi m \tilde{g}^{2}} \gg 1$, the order parameter can be approximated

$$
\Delta_{b} \approx 2 \sqrt{W \mu} \exp \left(-\frac{\epsilon_{0}-2 \mu}{2 \pi m \tilde{g}^{2}}\right)
$$

and a BCS superconductivity regime occurs where $\Delta_{b}$ is very small [62]. As a result, the boson density is very small $\left(\delta_{b} \ll\right.$ $\delta)$, which allows us to approximately solve for the chemical potential from Eq. (A14):

$$
\mu \approx \frac{\delta}{4 \pi m} .
$$

This regime is very similar to BCS superconductivity. This can be understood by integrating out the boson to obtain a 4fermion interaction $\tilde{g}^{\prime} c_{+}^{\dagger} c_{-}^{\dagger} c_{-} c_{+}$with $\tilde{g}^{\prime} \sim \frac{\tilde{g}^{2}}{\epsilon_{0}-2 \mu}$. In terms of $\tilde{g}^{\prime}$, the order parameter $\Delta_{b}$ scales exactly the same as the BCS order parameter (in two spatial dimensions): $\Delta_{b} \sim \Delta_{\mathrm{BCS}} \sim$ $\sqrt{W \mu} e^{-1 / D \tilde{g}^{\prime}}$, where $D=2 \pi m$ is the density of states from Eq. (A11).

Note that in this regime, the boson density is very small, so the $\tilde{g}$ coupling term in $H_{\text {eff }}$ [Eq. (A7)] contributes very little to the energy. Therefore, in this regime, the terms in the "..." of $H_{\text {eff }}$ are likely to play an important role and possibly result in other kinds of symmetry breaking. So although BCS-like superconductivity results when the "..." terms are dropped, a more detailed analysis is needed to determine the true ground state in this regime when the "..." terms are included.

\section{b. Resonantly paired superconductivity regime}

The boson density diverges as the chemical potential approaches its maximum value: $\mu \rightarrow \epsilon_{0} / 2$. Approximating $\mu \approx$ $\epsilon_{0} / 2$ allows us to solve for the boson density $\delta_{b}$ in Eqs. (A14), which can be used to express the order parameter [63]:

$$
\Delta_{b} \approx \tilde{g} \sqrt{\delta_{b}}, \quad \delta_{b} \approx \frac{1}{2} \delta-\pi m \epsilon_{0} .
$$




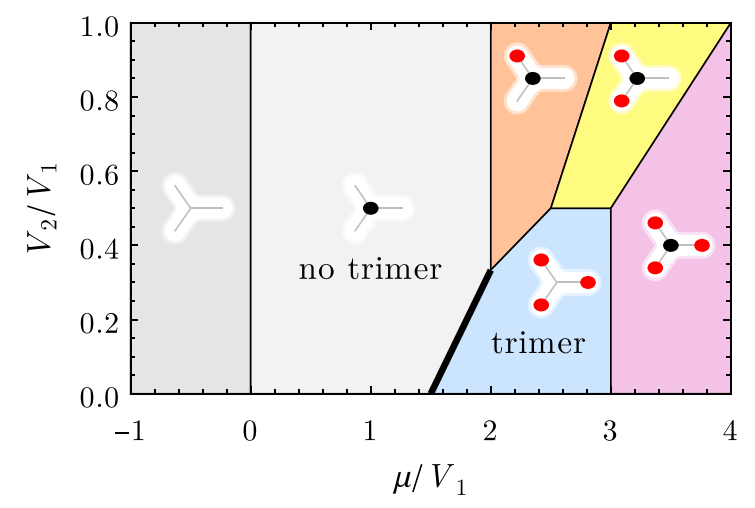

FIG. 9. Ground states of the four-site cluster model in Eq. (B1) with $\Delta=V_{1}$. Valence skipping (where charge of the ground state jumps by 2) [65] occurs along the thick line. Black and red dots denote filled orbitals with $n_{i}=1$.

Due to the significantly larger boson density, the order parameter $\Delta_{b} \sim \tilde{g}$ is immensely larger in this resonantly paired regime than in the BCS regime where $\Delta_{b} \sim e^{-1 / \tilde{g}^{2}}$ [Eq. (A15)].

\section{APPENDIX B: VALENCE SKIPPING IN A FOUR-SITE CLUSTER}

A toy model for trimer stability is obtained by considering a four-site cluster [64] of the Hamiltonian $H_{0}$ in Eq. (1) at a chemical potential $\mu$ :

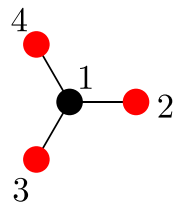

$$
\begin{aligned}
H_{4} & =\Delta\left(n_{2}+n_{3}+n_{4}\right) \\
& +V_{1} n_{1}\left(n_{2}+n_{3}+n_{4}\right) \\
& +V_{2}\left(n_{2} n_{3}+n_{3} n_{4}+n_{4} n_{2}\right) \\
& -\mu\left(n_{1}+n_{2}+n_{3}+n_{4}\right)
\end{aligned}
$$

Each site either has 0 or 1 fermions, $n_{i}=0,1$, which is physically relevant when a large on-site Hubbard interaction prevents double occupancy.

The ground-state phase diagram of the four-site cluster is shown in Fig. 9. The "no trimer" region is analogous to a change-transfer insulator, while the "trimer" region is analogous to a trimer excitation of the change-transfer insulator.

The ground states are easiest to understand in an ideal limit where $V_{2}=0, \Delta=V_{1}$, and $\mu=\frac{3}{2} V_{1}$. Then $H_{4}=V_{1}\left(n_{1}-\right.$ $\left.\frac{1}{2}\right)\left(n_{2}+n_{3}+n_{4}-\frac{3}{2}\right)$, and it is simple to see that the "no trimer" and "trimer" states are degenerate ground states.
[1] R. Bistritzer and A. H. MacDonald, Moiré bands in twisted double-layer graphene, Proc. Natl. Acad. Sci. U.S.A. 108, 12233 (2011).

[2] L. Balents, General continuum model for twisted bilayer graphene and arbitrary smooth deformations, SciPost Phys. 7, 048 (2019).

[3] J. M. B. Lopes dos Santos, N. M. R. Peres, and A. H. Castro Neto, Graphene Bilayer with a Twist: Electronic Structure, Phys. Rev. Lett. 99, 256802 (2007).

[4] Y. Cao, V. Fatemi, S. Fang, K. Watanabe, T. Taniguchi, E. Kaxiras, and P. Jarillo-Herrero, Unconventional superconductivity in magic-angle graphene superlattices, Nature (London) 556, 43 (2018).

[5] Y. Cao, V. Fatemi, A. Demir, S. Fang, S. L. Tomarken, J. Y. Luo, J. D. Sanchez-Yamagishi, K. Watanabe, T. Taniguchi, E. Kaxiras, R. C. Ashoori, and P. Jarillo-Herrero, Correlated insulator behaviour at half-filling in magic-angle graphene superlattices, Nature (London) 556, 80 (2018).

[6] X. Lu, P. Stepanov, W. Yang, M. Xie, M. A. Aamir, I. Das, C. Urgell, K. Watanabe, T. Taniguchi, G. Zhang, A. Bachtold, A. H. MacDonald, and D. K. Efetov, Superconductors, orbital magnets and correlated states in magic-angle bilayer graphene, Nature (London) 574, 653 (2019).

[7] G. Chen, L. Jiang, S. Wu, B. Lyu, H. Li, B. L. Chittari, K. Watanabe, T. Taniguchi, Z. Shi, J. Jung, Y. Zhang, and F. Wang, Evidence of a gate-tunable Mott insulator in a trilayer graphene moiré superlattice, Nat. Phys. 15, 237 (2019).

[8] Y. Choi, J. Kemmer, Y. Peng, A. Thomson, H. Arora, R. Polski, Y. Zhang, H. Ren, J. Alicea, G. Refael, F. von Oppen, K. Watanabe, T. Taniguchi, and S. Nadj-Perge, Electronic correlations in twisted bilayer graphene near the magic angle, Nat. Phys. 15, 1174 (2019).
[9] C. Shen, Y. Chu, Q. S. Wu, N. Li, S. Wang, Y. Zhao, J. Tang, J. Liu, J. Tian, K. Watanabe, T. Taniguchi, R. Yang, Z. Y. Meng, D. Shi, O. V. Yazyev, and G. Zhang, Correlated states in twisted double bilayer graphene, Nat. Phys. 16, 520 (2020).

[10] S. Carr, S. Fang, H. C. Po, A. Vishwanath, and E. Kaxiras, Derivation of Wannier orbitals and minimal-basis tight-binding Hamiltonians for twisted bilayer graphene: First-principles approach, Phys. Rev. Research 1, 033072 (2019).

[11] H. C. Po, L. Zou, T. Senthil, and A. Vishwanath, Faithful tightbinding models and fragile topology of magic-angle bilayer graphene, Phys. Rev. B 99, 195455 (2019).

[12] M. Koshino, N. F. Q. Yuan, T. Koretsune, M. Ochi, K. Kuroki, and L. Fu, Maximally Localized Wannier Orbitals and the Extended Hubbard Model for Twisted Bilayer Graphene, Phys. Rev. X 8, 031087 (2018).

[13] J. Kang and O. Vafek, Symmetry, Maximally Localized Wannier States, and a Low-Energy Model for Twisted Bilayer Graphene Narrow Bands, Phys. Rev. X 8, 031088 (2018).

[14] G. Tarnopolsky, A. J. Kruchkov, and A. Vishwanath, Origin of Magic Angles in Twisted Bilayer Graphene, Phys. Rev. Lett. 122, 106405 (2019).

[15] X.-C. Wu, C.-M. Jian, and C. Xu, Coupled-wire description of the correlated physics in twisted bilayer graphene, Phys. Rev. B 99, 161405(R) (2019).

[16] S. Manzeli, D. Ovchinnikov, D. Pasquier, O. V. Yazyev, and A. Kis, 2D transition metal dichalcogenides, Nat. Rev. Mater. 2, 17033 (2017).

[17] L. Wang, E.-M. Shih, A. Ghiotto, L. Xian, D. A. Rhodes, C. Tan, M. Claassen, D. M. Kennes, Y. Bai, B. Kim, K. Watanabe, T. Taniguchi, X. Zhu, J. Hone, A. Rubio, A. Pasupathy, and C. R. Dean, Correlated electronic phases in twisted bilayer transition metal dichalcogenides, Nat. Mater. 19, 861 (2020). 
[18] Q. Shi, E.-M. Shih, M. V. Gustafsson, D. A. Rhodes, B. Kim, K. Watanabe, T. Taniguchi, Z. Papić, J. Hone, and C. R. Dean, Odd- and even-denominator fractional quantum Hall states in monolayer $\mathrm{WSe}_{2}$, Nat. Nanotechnol. 15, 569 (2020).

[19] J. Wang, Q. Shi, E.-M. Shih, L. Zhou, W. Wu, Y. Bai, D. A. Rhodes, K. Barmak, J. Hone, C. R. Dean, and X. Y. Zhu, Excitonic phase transitions in $\mathrm{MoSe}_{2} / \mathrm{WSe}_{2}$ heterobilayers, arXiv:2001.03812.

[20] C. Jin, E. C. Regan, A. Yan, M. Iqbal Bakti Utama, D. Wang, S. Zhao, Y. Qin, S. Yang, Z. Zheng, S. Shi, K. Watanabe, T. Taniguchi, S. Tongay, A. Zettl, and F. Wang, Observation of moiré excitons in $\mathrm{WSe}_{2} / \mathrm{WS}_{2}$ heterostructure superlattices, Nature (London) 567, 76 (2019).

[21] Y. Shimazaki, I. Schwartz, K. Watanabe, T. Taniguchi, M. Kroner, and A. Imamoğlu, Strongly correlated electrons and hybrid excitons in a moiré heterostructure, Nature 580, 472 (2020).

[22] J. Sung, Y. Zhou, G. Scuri, V. Zólyomi, T. I. Andersen, H. Yoo, D. S. Wild, A. Y. Joe, R. J. Gelly, H. Heo, S. J. Magorrian, D. Bérubé, A. M. M. Valdivia, T. Taniguchi, K. Watanabe, M. D. Lukin, P. Kim, V. I. Fal'ko, and H. Park, Broken mirror symmetry in excitonic response of reconstructed domains in twisted $\mathrm{MoSe}_{2} / \mathrm{MoSe}_{2}$ bilayers, Nat. Nanotechnol. 15, 750 (2020).

[23] Z. Bi and L. Fu, Excitonic density wave and spin-valley superfluid in bilayer transition metal dichalcogenide, Nat. Commun. (to be published), arXiv:1911.04493.

[24] F. Wu, T. Lovorn, E. Tutuc, and A. H. MacDonald, Hubbard Model Physics in Transition Metal Dichalcogenide Moiré Bands, Phys. Rev. Lett. 121, 026402 (2018).

[25] F. Wu, T. Lovorn, E. Tutuc, I. Martin, and A. H. MacDonald, Topological Insulators in Twisted Transition Metal Dichalcogenide Homobilayers, Phys. Rev. Lett. 122, 086402 (2019).

[26] L. Xian, M. Claassen, D. Kiese, M. M. Scherer, S. Trebst, D. M. Kennes, and A. Rubio, Realization of nearly dispersionless bands with strong orbital anisotropy from destructive interference in twisted bilayer $\mathrm{MoS}_{2}$, arXiv:2004.02964.

[27] Y. Tang, L. Li, T. Li, Y. Xu, S. Liu, K. Barmak, K. Watanabe, T. Taniguchi, A. H. MacDonald, J. Shan, and K. F. Mak, Simulation of Hubbard model physics in $\mathrm{WSe}_{2} / \mathrm{WS}_{2}$ moiré superlattices, Nature 579, 353 (2020).

[28] E. C. Regan, D. Wang, C. Jin, M. I. Bakti Utama, B. Gao, X. Wei, S. Zhao, W. Zhao, K. Yumigeta, M. Blei, J. Carlstroem, K. Watanabe, T. Taniguchi, S. Tongay, M. Crommie, A. Zettl, and F. Wang, Mott and generalized Wigner crystal states in $\mathrm{WSe}_{2} / \mathrm{WS}_{2}$ moiré superlattices, Nature 579, 359 (2020).

[29] Y. Xu, S. Liu, D. A. Rhodes, K. Watanabe, T. Taniguchi, J. Hone, V. Elser, K. F. Mak, and J. Shan, Abundance of correlated insulating states at fractional fillings of $\mathrm{WSe}_{2} / \mathrm{WS}_{2}$ moiré superlattices, Nature 587, 214 (2020).

[30] Y. Zhang, N. F. Q. Yuan, and L. Fu, Moiré quantum chemistry: Charge transfer in transition metal dichalcogenide superlattices, arXiv:1910.14061.

[31] Localized and symmetric Wannier orbitals are not even possible in twisted bilayer graphene [11].

[32] J. Zaanen, G. A. Sawatzky, and J. W. Allen, Band Gaps and Electronic Structure of Transition-Metal Compounds, Phys. Rev. Lett. 55, 418 (1985).

[33] V. J. Emery, Theory of High- $\mathrm{T}_{\mathrm{c}}$ Superconductivity in Oxides, Phys. Rev. Lett. 58, 2794 (1987)
[34] F. C. Zhang and T. M. Rice, Effective Hamiltonian for the superconducting Cu oxides, Phys. Rev. B 37, 3759 (1988).

[35] Y. Bar-Yam, Two-component superconductivity. I. Introduction and phenomenology, Phys. Rev. B 43, 359 (1991).

[36] E. Abrahams, S. Schmitt-Rink, and C. M. Varma, High$\mathrm{T}_{c}$ superconductivity mediated by charge transfer excitations, Physica B+C 148, 257 (1987).

[37] M. Grilli, R. Raimondi, C. Castellani, C. Di Castro, and G. Kotliar, Superconductivity, Phase Separation, and ChargeTransfer Instability in the $U=\infty$ Limit of the Three-Band Model of the $\mathrm{CuO}_{2}$ Planes, Phys. Rev. Lett. 67, 259 (1991).

[38] V. Gurarie and L. Radzihovsky, Resonantly paired fermionic superfluids, Ann. Phys. 322, 2 (2007).

[39] Q. Chen, J. Stajic, S. Tan, and K. Levin, BCS-BEC crossover: From high temperature superconductors to ultracold superfluids, Phys. Rep. 412, 1 (2005).

[40] T. Liu and Y. Zhang (private communication). Calculated using the screened Coulomb interaction shown in Eq. (A3) and the resulting localized Wannier orbitals.

[41] $V_{c}=3 V_{1}+3 V_{3}+6 V_{4}+\cdots$ and $V_{a}=6 V_{2}+6 V_{5}+\cdots$, where $V_{n}$ is the repulsion between $n$ th-nearest neighbors, shown in Fig. 4.

[42] S. R. White, S. Chakravarty, M. P. Gelfand, and S. A. Kivelson, Pair binding in small Hubbard-model molecules, Phys. Rev. B 45, 5062 (1992).

[43] H. Yao, W.-F. Tsai, and S. A. Kivelson, Myriad phases of the checkerboard Hubbard model, Phys. Rev. B 76, 161104(R) (2007).

[44] L. Isaev, G. Ortiz, and C. D. Batista, Superconductivity in Strongly Repulsive Fermions: The Role of Kinetic-Energy Frustration, Phys. Rev. Lett. 105, 187002 (2010).

[45] K. Slagle and Y. B. Kim, A simple mechanism for unconventional superconductivity in a repulsive fermion model, SciPost Phys. 6, 016 (2019).

[46] B. Padhi, C. Setty, and P. W. Phillips, Doped twisted bilayer graphene near magic angles: Proximity to Wigner crystallization, not Mott insulation, Nano Lett. 18, 6175 (2018).

[47] J. Hubbard, Generalized Wigner lattices in one dimension and some applications to tetracyanoquinodimethane (TCNQ) salts, Phys. Rev. B 17, 494 (1978).

[48] L. Rademaker, Y. Pramudya, J. Zaanen, and V. Dobrosavljević, Influence of long-range interactions on charge ordering phenomena on a square lattice, Phys. Rev. E 88, 032121 (2013).

[49] K. Jerzy Kapcia, J. Barański, and A. Ptok, Diversity of charge orderings in correlated systems, Phys. Rev. E 96, 042104 (2017).

[50] H.-D. Chen, O. Vafek, A. Yazdani, and S.-C. Zhang, Pair Density Wave in the Pseudogap State of High Temperature Superconductors, Phys. Rev. Lett. 93, 187002 (2004).

[51] See, e.g., Appendix B.1 of Ref. [38].

[52] Y. Zhang, T. Liu, and L. Fu, Electrically tunable charge transfer and charge orders in twisted transition metal dichalcogenide bilayers, arXiv:2009.14224.

[53] M. Angeli and A. H. MacDonald, $\Gamma$-valley transition-metaldichalcogenide moirè bands, arXiv:2008.01735.

[54] H. Li, S. Li, M. H. Naik, J. Xie, X. Li, J. Wang, E. Regan, D. Wang, W. Zhao, S. Zhao, S. Kahn, K. Yumigeta, M. Blei, T. Taniguchi, K. Watanabe, S. Tongay, A. Zettl, S. G. Louie, F. Wang, and M. F. Crommie, Imaging moiré flat bands in 3D reconstructed $\mathrm{WSe}_{2} / \mathrm{WS}_{2}$ superlattices, arXiv:2007.06113. 
[55] L. Rademaker and P. Mellado, Charge-transfer insulation in twisted bilayer graphene, Phys. Rev. B 98, 235158 (2018).

[56] F. Guinea and N. R. Walet, Electrostatic effects, band distortions, and superconductivity in twisted graphene bilayers, Proc. Natl. Acad. Sci. U.S.A. 115, 13174 (2018).

[57] X. Liu, Z. Hao, E. Khalaf, J. Y. Lee, K. Watanabe, T. Taniguchi, A. Vishwanath, and P. Kim, Spin-polarized correlated insulator and superconductor in twisted double bilayer graphene, Nature 583, 221 (2020).

[58] G. Chen, A. L. Sharpe, P. Gallagher, I. T. Rosen, E. Fox, L. Jiang, B. Lyu, H. Li, K. Watanabe, T. Taniguchi, J. Jung, Z. Shi, D. Goldhaber-Gordon, Y. Zhang, and F. Wang, Signatures of tunable superconductivity in a trilayer graphene moiré superlattice, Nature 572, 215 (2019).

[59] N. Luo, Variational bounds on the ground-state energy of three electrons and one hole in two-dimension, arXiv:condmat/0101004.

[60] A single parallel conductor results in $V(r) \propto \frac{1}{r}-$ $1 / \sqrt{r^{2}+(2 d)^{2}}$.
[61] The perturbative approximation $\tilde{g} \sim t_{1} t_{2} / V_{2}$ formally also requires assuming $V_{4} \ll V_{2}$ so that $V_{4}$ only results in a perturbative correction to the final state energy in Fig. 3(b).

[62] The $\sqrt{W \mu}$ prefactor in $\Delta_{b}$ [in Eq. (A15)] comes from the limits of integration $(-\mu$ to $W)$ in Eq. (A11). Equation (A15) is only valid when $\mu>0$. If $\mu=0$, then note that the ground-state energy in the $\mu=0$ limit is equivalent to the energy in the $\mu=W$ limit if the mass is halved; i.e., $\left.E_{\mathrm{MF}}\right|_{\mu=0}=\left.E_{\mathrm{MF}}\right|_{\mu=W} ^{m \rightarrow m / 2}$ in Eq. (A12). Therefore, if $\mu=0$, then $\Delta_{b} \approx 2 W \exp -\left(-\frac{\epsilon_{0}-2 \mu}{\pi m \tilde{g}^{2}}\right)$ [by replacing $\mu \rightarrow W$ and $m \rightarrow m / 2$ in Eq. (A15)], which is significantly smaller than the expression in Eq. (A15) when $0<\mu$ and $\frac{\epsilon_{0}-2 \mu}{\pi m \tilde{g}^{2}} \gg 1$ due to the missing factor of $\frac{1}{2}$ in the exponent.

[63] A similar equation for the boson density in three spatial dimensions appears in Eq. (6.8) of Ref. [38].

[64] M. E. Raikh, L. I. Glazman, and L. E. Zhukov, Two-Electron State in a Disordered 2D Island: Pairing Caused by the Coulomb Repulsion, Phys. Rev. Lett. 77, 1354 (1996).

[65] C. M. Varma, Missing Valence States, Diamagnetic Insulators, and Superconductors, Phys. Rev. Lett. 61, 2713 (1988). 